\title{
Self-Adaptive Gas Sensor System based on Operating Conditions using Data Prediction
}

Kyusung Kim ${ }^{1}$, Phuwadej Pornaroontham ${ }^{2}$, Pil gyu Choi ${ }^{1}$, Toshio Itoh ${ }^{1}$, Yoshitake

$$
\operatorname{Masuda}^{1 *}
$$

${ }^{1}$ National Institute of Advanced Industrial Science and Technology (AIST), 2266-98

Anagahora, Shimoshidami, Moriyama, Nagoya 463-8560, Japan

${ }^{2}$ Department of Chemical Systems Engineering, Graduate School of Engineering,

Nagoya University, Nagoya 464-8603, Japan 
Table S1. Various types of $\mathrm{SnO}_{2}$-based acetone gas sensors with their responses for specific concentrations.

\begin{tabular}{|c|c|c|c|}
\hline Sensing material & Response & Concentration & Reference \\
\hline $\mathrm{SnO}_{2}$ microsphere with $\mathrm{Au}$ and $\mathrm{NiO}$ & 5.0 & $1000 \mathrm{ppb}$ & {$[31]$} \\
\hline Ru-doped $\mathrm{SnO}_{2}$ nanofibers & 8.9 & $5000 \mathrm{ppb}$ & {$[32]$} \\
\hline Mesoporous $\mathrm{SnO}_{2}$ & 1.5 & $1000 \mathrm{ppb}$ & {$[33]$} \\
\hline Co-catalyzed $\mathrm{SnO}_{2}$ nanosphere & 1.8 & $1000 \mathrm{ppb}$ & {$[34]$} \\
\hline $\mathrm{SnO}_{2}$ nanowire with $\mathrm{Co}_{3} \mathrm{O}_{4}$ nanoparticles & 4.0 & $1000 \mathrm{ppb}$ & {$[35]$} \\
\hline Nanotubular $\mathrm{SnO}_{2}$ & 1.6 & $1000 \mathrm{ppb}$ & {$[36]$} \\
\hline Pt-doped 3D porous $\mathrm{SnO}_{2}$ & 2.1 & $50 \mathrm{ppb}$ & {$[37]$} \\
\hline $\mathrm{Fe}_{2} \mathrm{O}_{3} / \mathrm{SnO}_{2}$ Nanoball & 4.0 & $50 \mathrm{ppb}$ & {$[38]$} \\
\hline \multirow{2}{*}{$\mathrm{SnO}_{2}$ nanosheet } & 5.8 & $50 \mathrm{ppb}$ & \multirow{2}{*}{ Present work } \\
\hline & 15.4 & $1000 \mathrm{ppb}$ & \\
\hline
\end{tabular}


Table S2. Analysis of variance (ANOVA) of the terms for the $2^{3}$ factorial design with center points.

(a) without curvature

\begin{tabular}{cccccc}
\hline Source & Sum of squares & $\mathrm{df}^{\mathrm{a}}$ & Mean squares & F-value & p-value \\
\hline Model & 171.06 & 6 & 28.51 & 0.85 & 0.5801 \\
A-Temperature & 1.92 & 1 & 1.92 & 0.06 & 0.8199 \\
B-Gas flow & 3.89 & 1 & 3.89 & 0.12 & 0.7466 \\
C-Concentration & 161.64 & 1 & 161.64 & 4.85 & 0.0790 \\
AB & 0.17 & 1 & 0.17 & 0.01 & 0.9461 \\
AC & 0.31 & 1 & 0.31 & 0.01 & 0.9267 \\
BC & 3.13 & 1 & 3.13 & 0.09 & 0.7719 \\
Residual & 166.81 & 5 & 33.36 & - & - \\
Lack of Fit & 160.69 & 2 & 80.35 & 39.42 & 0.0070 \\
Pure Error & 6.11 & 3 & 2.04 & - & - \\
Cor Total & 337.86 & 11 & & - & - \\
Std. Dev. & 5.78 & & Adjusted R ${ }^{2}$ & -0.0862 & \\
Mean $_{\text {C.V. } \% \text { c }}$ & 10.86 & & Predicted R & -6.0456 & \\
BIC & 53.20 & & Adeq Precision & 2.5763 & \\
\hline
\end{tabular}

aDegree of freedom

bStandard of deviation

${ }^{c}$ Coefficient of variation

dBayesian information criterion

eAdequate precision 
(b) with curvature

\begin{tabular}{|c|c|c|c|c|c|}
\hline Source & Sum of squares & $\mathrm{df}^{\mathrm{a}}$ & Mean squares & F-value & p-value \\
\hline Model & 171.06 & 6 & 28.51 & 5.76 & 0.0559 \\
\hline A-Temperature & 1.92 & 1 & 1.92 & 0.39 & 0.5670 \\
\hline B-Gas flow & 3.89 & 1 & 3.89 & 0.79 & 0.4252 \\
\hline C-Concentration & 161.64 & 1 & 161.64 & 32.67 & 0.0046 \\
\hline $\mathrm{AB}$ & 0.17 & 1 & 0.17 & 0.03 & 0.8627 \\
\hline $\mathrm{AC}$ & 0.31 & 1 & 0.31 & 0.06 & 0.8141 \\
\hline $\mathrm{BC}$ & 3.13 & 1 & 3.13 & 0.63 & 0.4713 \\
\hline Curvature & 147.02 & 1 & 147.02 & 29.71 & 0.0055 \\
\hline Residual & 19.79 & 4 & 4.95 & - & - \\
\hline Lack of Fit & 13.68 & 1 & 13.68 & 6.71 & 0.0810 \\
\hline Pure Error & 6.11 & 3 & 2.04 & - & - \\
\hline Cor Total & 337.86 & 11 & & & \\
\hline Std. Dev. ${ }^{b}$ & 2.22 & & $\mathrm{R}^{2}$ & 0.8963 & \\
\hline Mean & 10.86 & & Adjusted $\mathrm{R}^{2}$ & 0.7408 & \\
\hline C.V. $\%$ c & 20.49 & & Predicted $\mathrm{R}^{2}$ & -3.6433 & \\
\hline $\mathrm{BIC}^{\mathrm{d}}$ & 57.45 & & Adeq Precision ${ }^{\mathrm{e}}$ & 7.0616 & \\
\hline
\end{tabular}

${ }^{a}$ Degree of freedom

bStandard of deviation

${ }^{\mathrm{c} C o e f f i c i e n t}$ of variation

${ }^{\mathrm{d}}$ Bayesian information criterion

${ }^{\mathrm{e}}$ Adequate precision 
Table S3. Analysis of variance (ANOVA) of the terms for full model by FCCCM-RSM.

\begin{tabular}{|c|c|c|c|c|c|}
\hline Source & Sum of squares & $d f^{a}$ & Mean squares & F-value & p-value \\
\hline Block & 45.72 & 1 & 45.72 & - & - \\
\hline Model & 426.01 & 9 & 47.33 & 13.27 & 0.0003 \\
\hline A-Temperature & 0.45 & 1 & 0.45 & 0.12 & 0.7320 \\
\hline B-Gas flow & 11.09 & 1 & 11.09 & 3.11 & 0.1117 \\
\hline C-Concentration & 228.10 & 1 & 228.10 & 63.94 & $<0.0001$ \\
\hline $\mathrm{AB}$ & 0.17 & 1 & 0.17 & 0.05 & 0.8329 \\
\hline $\mathrm{AC}$ & 0.31 & 1 & 0.31 & 0.09 & 0.7741 \\
\hline $\mathrm{BC}$ & 3.13 & 1 & 3.13 & 0.88 & 0.3737 \\
\hline $\mathrm{A}^{2}$ & 85.28 & 1 & 85.28 & 23.91 & 0.0009 \\
\hline $\mathrm{B}^{2}$ & 0.11 & 1 & 0.11 & 0.03 & 0.8617 \\
\hline $\mathrm{C}^{2}$ & 5.58 & 1 & 5.58 & 1.56 & 0.2426 \\
\hline Residual & 32.11 & 9 & 3.57 & - & - \\
\hline Lack of Fit & 25.89 & 5 & 5.18 & 3.33 & 0.1334 \\
\hline Pure Error & 6.22 & 4 & 1.55 & - & - \\
\hline Cor Total & 503.84 & 19 & - & - & - \\
\hline Std. Dev. ${ }^{b}$ & 1.89 & & $\mathrm{R}^{2}$ & 0.9299 & \\
\hline Mean & 12.09 & & Adjusted R² & 0.8598 & \\
\hline C.V. $\%{ }^{c}$ & 15.62 & & Predicted $\mathrm{R}^{2}$ & 0.0197 & \\
\hline $\mathrm{BIC}^{\mathrm{d}}$ & 99.18 & & Adeq Precision ${ }^{\mathrm{e}}$ & 11.7276 & \\
\hline
\end{tabular}

aDegree of freedom

${ }^{\mathrm{b}}$ Standard of deviation

${ }^{c}$ Coefficient of variation

${ }^{\mathrm{d}}$ Bayesian information criterion

eAdequate precision 
Table S4. Analysis of variance (ANOVA) of the terms for reduced models by stepwise regression via (a) p-value and (b) BIC criteria.

(a) RM-1

\begin{tabular}{cccccc}
\hline Source & Sum of squares & $\mathrm{df}^{\mathrm{a}}$ & Mean squares & F-value & p-value \\
\hline Block & 45.72 & 1 & 45.72 & - & - \\
Model & 404.25 & 3 & 134.75 & 37.52 & $<0.0001$ \\
A-Temperature & 0.45 & 1 & 0.45 & 0.12 & 0.7297 \\
C-Concentration & 228.10 & 1 & 228.10 & 63.52 & $<0.0001$ \\
$\mathrm{~A}^{2}$ & 175.71 & 1 & 175.71 & 48.93 & $<0.0001$ \\
Residual & 53.87 & 15 & 3.59 & - & - \\
Lack of Fit & 47.65 & 11 & 4.33 & 2.79 & 0.1671 \\
Pure Error & 6.22 & 4 & 1.55 & - & - \\
Cor Total & 503.84 & 19 & - & - & - \\
Std. Dev. $^{\mathrm{b}}$ & 1.90 & & $\mathrm{R}^{2}$ & 0.8824 & \\
Mean $_{\text {C.V. } \%{ }^{\mathrm{c}}}$ & 12.09 & & Adjusted R & 0.8589 & \\
BIC $^{\mathrm{d}}$ & 15.67 & & Predicted R & 0.7830 & \\
\hline
\end{tabular}

aDegree of freedom

bStandard of deviation

coefficient of variation

${ }^{\mathrm{d} B a y e s i a n}$ information criterion

eAdequate precision 
(b) RM-2

\begin{tabular}{|c|c|c|c|c|c|}
\hline Source & Sum of squares & $d f^{a}$ & Mean squares & F-value & p-value \\
\hline Block & 45.72 & 1 & 45.72 & & \\
\hline Model & 422.29 & 5 & 84.46 & 30.65 & $<0.0001$ \\
\hline A-Temperature & 0.45 & 1 & 0.45 & 0.16 & 0.6943 \\
\hline B-Gas flow & 11.09 & 1 & 11.09 & 4.02 & 0.0661 \\
\hline C-Concentration & 228.10 & 1 & 228.10 & 82.77 & $<0.0001$ \\
\hline $\mathrm{A}^{2}$ & 99.03 & 1 & 99.03 & 35.93 & $<0.0001$ \\
\hline $\mathrm{C}^{2}$ & 6.95 & 1 & 6.95 & 2.52 & 0.1362 \\
\hline Residual & 35.83 & 13 & 2.76 & - & - \\
\hline Lack of Fit & 29.61 & 9 & 3.29 & 2.12 & 0.2445 \\
\hline Pure Error & 6.22 & 4 & 1.55 & - & - \\
\hline Cor Total & 503.84 & 19 & & - & - \\
\hline Std. Dev. ${ }^{b}$ & 1.66 & & $\mathrm{R}^{2}$ & 0.9218 & \\
\hline Mean & 12.09 & & Adjusted $\mathrm{R}^{2}$ & 0.8917 & \\
\hline C.V. $\%{ }^{c}$ & 13.73 & & Predicted $\mathrm{R}^{2}$ & 0.7882 & \\
\hline $\mathrm{BIC}^{\mathrm{d}}$ & 89.39 & & Adeq Precision ${ }^{\mathrm{e}}$ & 16.9034 & \\
\hline
\end{tabular}

aDegree of freedom

bStandard of deviation

${ }^{c}$ Coefficient of variation

${ }^{\mathrm{d}}$ Bayesian information criterion

eAdequate precision 



Fig. S1. Diagnostic plots for RM-2 model including (a) normal plot of residuals, (b) residuals versus predicted plot, (c) residuals versus run plot and (d) plot of residuals versus blocks. 